\title{
Assessment of the Contribution of Time Reversal on a UWB Localization System for Railway Applications
}

\author{
Bouna Fall • Fouzia Elbahhar • Marc Heddebaut • \\ Atika Rivenq • Maria Gabriella Di Benedetto
}

Received: 21 January 2014 /Revised: 18 November 2014 / Accepted: 21 November 2014

(C) Springer Science+Business Media New York 2014

\begin{abstract}
UWB radio has the potential to offer good performance in terms of localization precision. Time Reversal channel pre-filtering facilitates signal detection and also helps to increase the received energy in a targeted area. This paper assesses the quantitative and qualitative contributions of the Time Reversal (TR) technique associated with an Ultra Wideband (UWB) localization system applied to a railway odometry problem. The analytical and simulation results for Power Delay Profile, equivalent channel model and focusing gain of the TRUWB are given. The contribution of the TR technique associated with UWB radio to enhance the localization resolution is analysed. To perform these studies, and to be representative of the proposed railway application, a deterministic channel model is used. The analytical simulation and experimental results show that time reversal has very promising characteristics regarding its association with the UWB in terms of localization error. These results also indicate that the TR-UWB technique delivers improved performance over UWB only localization approach, that could benefit the development of the proposed railway application.
\end{abstract}

Keywords Ultra wide band (UWB) · Time reversal (TR) . TDOA · IEEE 802.15.3a channel models · Temporal focusing $\cdot$ Spatial focusing

B. Fall $(\bowtie) \cdot$ A. Rivenq

University, Valenciennes, France

e-mail: bouna.fall@univ-valenciennes.fr

F. Elbahhar $\cdot$ M. Heddebaut

IFSTTAR/LEOST, Villeneuve d'Ascq, France

M. G. Di Benedetto

University, University, Italia

\section{Introduction}

Railway transport is progressing significantly nowadays, providing many benefits. To achieve an efficient and safe train control and command system, it is essential to ensure an adequate exchange of information between trains and the ground. It is also necessary to accurately know the localization of all trains along the tracks [1]. This can be performed using a continuous coverage radio communication and localization system [2]. Otherwise, signalling systems relying on the use of balises located on the ground are also widely used. A balise is a piece of electronic equipment providing track to train spot communication when the train passes over it [3, 4]. Balises are bolt on ties and corresponding balise-readers are installed beneath the trains. The reader communicates with the balise while the train passes over the balise, exchanging data with the infrastructure. Balises are georeferenced and are also railway kilometre markers. They provide absolute localization information to the trains. Balise communication range is limited, usually less than one metre. Therefore, in balise-based signalling systems, communication and absolute localization information between trains and ground are only provided at discrete, consecutive locations along the track [4]. Consequently, especially at high speed, high data rate communication is employed to exchange all the necessary data in a limited communication time. On board the train, a continuous localization process is run based on data provided by the train proprioceptive sensors [5]. Usually, these sensors are phonic wheels and Doppler radars. They are used for relative distance and speed measurements. When passing over a balise, the train receives the localization information sent by the balise and determines its absolute localization. This information is used to compensate the drift of the relative distance measurement based on the train sensors only. Usually, balises are spaced every $2-3 \mathrm{~km}$ along the track in order to limit the localization drift to a tolerable safe value. Shorter spacing is used in the vicinity of railway stations or shunting areas. 
This study concentrates on the localization problem by proposing a more precise localization and less bulky system using new approach based on the Ultra-wideband (UWB) technology and Time Reversal (TR) technique. The short UWB pulses are ideal candidates for indoor localization applications. Their short duration assures the resistance against multipath effects and gives a good time resolution. Because of the fact that the UWB has a very strict power emission limits, the short-range (e.g., indoor) applications are aimed for [6]. Due to reciprocity, the TR technique offers a better temporal and spatial focusing resolution as compared to the conventional techniques which fail to achieve the desired resolution due to several problems such as, the difficulties of signal detection at the reception and the limited antenna aperture [7]. Thus, when the UWB is associated with TR technique, it can perform superior localization even when the targets are very close to each other. It evaluates the characteristics of TR including temporal focusing and spatial focusing by using a deterministic channel model. For this evaluation, theoretical, simulation and experimental methods are used. This work mainly aims at verifying whether the combination of UWB and TR techniques may allow for the railway decimetre to be obtained with the necessary level of precision.

The remainder of this paper is organized as follows. Section 2 presents new proposal system and describes the UWB, the TR techniques and the deterministic channel model. Section 3 gives the analytical study of TR characteristics (temporal focusing and spatial focusing) and performance evaluation of TR-UWB systems in terms of localization error. Experimental results of TR characteristics and localization error evaluation are presented in section 4. Finally, conclusions and future work are provided in section 5.

\section{System Description}

\subsection{New Balise Proposal}

Conventionally the balise, located between the rails, has a form of a rectangular parallelepiped as shown in Fig. 1. The train, passing over the balise, exchanges information at high rate with the ground, reads its absolute location and compensates for the drift of its proprioceptive localization sensors.

In many railway systems, these balises constitute the only equipment remaining on the track, between the rails [8]. Therefore, it could prove worthwhile to remove this remaining equipment from the track in order to facilitate track maintenance. Indeed the corrective error beacon systems (wheel wear, skate phenomena and jamming or the presence of water on the road constitute cumulative error factors) are disposed on the ground and raises unreliable stresses during maintenance of the track. As presented in Fig. 1, the conventional balise situated between the rails could be replaced by a new proposed balise, installed on a pole, on the side of the track and a few meters away. This balise allows increasing the resolution. There are several scenarios for which the resolution needs to be improved, for example:

- Specific vehicles stop (subway) before landing doors (location);

- $\quad$ stopped the train or subway in front of a space reserved for persons with disabilities and with reduced mobility;

- Stop the vehicle (train) after alert to possible dangers (location / communication).

Therefore, this new balise does not interfere anymore with track maintenance operations i.e. rail replacements.

It uses an Ultra Wideband Impulse-Radio technique (UWBIR) associated with a TR technique. The UWB-IR radio technique is used to support high data rate ground to train communication as well as the train localization process. The TR technique helps focusing balise radiation on a small area over the track, represented by a sphere in Fig. 1. When crossing this focusing area, the train antenna located in front of the train receives these UWB signals and computes its position to the geo-referenced balise using time of flight information. In the scope of TR-UWB system, the target accuracy is less than $10 \mathrm{~cm}$ with a maximum range of $50 \mathrm{~m}$.

Beyond the railway system, the combination of the techniques may find application in various areas above, such as the detection and localization of persons-through barriers and finding victims of accidents, especially in the mountains or in mines [9]. Through-the-Wall sensing also takes advantage of TR techniques; this application is very useful in safety and peace-keeping applications. Otherwise TR technique focuses the radiation from the balise in a small area between the rails (e.g., at or are currently placed KVB balises). This focusing concentrates the energy in time and space, allowing, with the combination of UWB technology, calculating with good accuracy and good resolution its position relative to the beacon geo-referenced.

\subsection{Ultra Wideband (UWB) Technology}

UWB radio is typically defined as a wireless transmission scheme with a bandwidth of over $500 \mathrm{MHz}$, or having a fractional bandwidth higher than 0.2 [10]. There are basely two ways of obtaining an UWB signals: OFDM and impulse radio UWB approaches. In our study, the second method is chosen. This involves the transmission of very short pulses occupying a very wide spectrum. The Gaussian waveform pulse or its derivative is commonly used pulse shape.

Localization in indoor environments is subject to two major sources of error, the first being the lack of line of sight (LOS) between the transmitter and the receiver, and the second being the excessive presence of multipath $[11,12]$. The introduction of UWB in wireless communications, performance under this aspect may improve. Previous investigations have raised, however, major issues, such as the complexity of 
Fig. 1 TR-UWB proposed

localization system

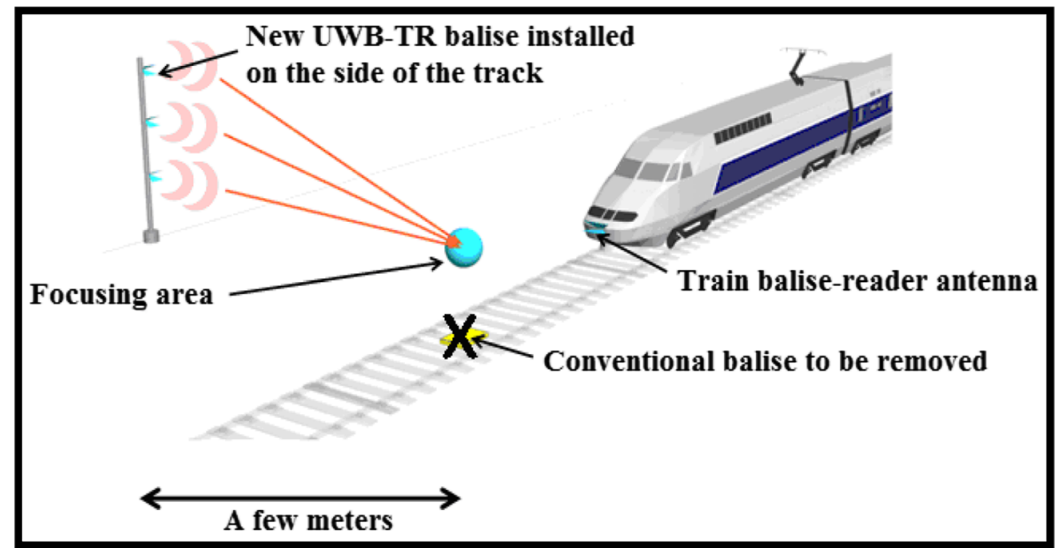

the signal processing at the reception $[13,14]$. UWB has been associated with TR technique, especially in multi-users communication systems [15], in order to assess the above problems.

\subsection{Time Reversal Technique}

Classically, Time Reversal (TR) has been applied to acoustics and underwater systems $[16,17]$. It is closely related to the retrodirective array in microwave $[18,19]$ and phase conjugation in optics. The first TR experiment using electromagnetic waves in the $2.45 \mathrm{GHz}$ band was reported in [20]. This contribution suggests that the techniques developed for ultrasound might also be used for the study of electromagnetic case. It is an interesting challenge because in many real environments (buildings or cities), microwaves, using wavelengths between 5 and $30 \mathrm{~cm}$, are scattered off by objects such as walls, desks, vehicles and so on, which produces a multitude of paths from the transmitter to the receiver.

In such situations, a TR system should be able not only to compensate for the multipath effect, but also to improve radio communication parameters, thanks to the many reflections/ reverberations. TR has two main characteristics, the temporal focusing and the spatial focusing; these are very beneficial to the UWB system [21, 22]. More recently, TR has also been studied for broadband especially for UWB communications [23].

In the proposed TR-UWB system, the signal shape to transmit is selected as the second Gaussian derivative function. In addition, the channel impulse response (CIR) is measured between the transmitter $(\mathrm{Tx})$ and the receiver $(\mathrm{Rx})$ and the channel state information is loaded into Tx. Thirdly, the selected signal and the impulse response are reversed in time and transmitted by $\mathrm{Tx}$ in the propagation channel, up to Rx. As represented in Fig. 2, this can be mathematically described by noting $s(t)$ the transmitted pulse, $h(t)$ the complex impulse response of the channel and $h$ $*(-t)$ the complex conjugate of the time reversed version of $h(t)$; $y(t)$ the received signal without TR and $y_{R T}(t)$, the received signal with TR at the receiver; One has:

$y(t)=s(t) \otimes h(t)+n(t)$

$y_{R T}(t)=s(t) \otimes h^{*}(-t) \otimes h(t)+n(t)$

Where $\otimes$ represents the convolution operation and $n(t)$ is the Gaussian noise.

From Eq. (2), we deduce the equivalent impulse response $h e q(t)$ which corresponds to the autocorrelation function of the channel [21]:

$h e q(t)=h^{*}(-t) \otimes h(t)$

The local Channel State Information (CSI) between any balise transmitter and the distant area, between the rails, where the energy must be focused is measured or computed a single time during the installation phase of the balise. This CSI information is then loaded in the transmitter equipment to perform the TR operation. As long as the propagation environment remains unmodified, this initial CSI is repetitively used by the balise. This information is then introduced as prefiltering data in the different UWB transmitters. Therefore, focusing is obtained in the required position, along the track, potentially improving the absolute localization process [24]. Moreover using a single balise, focusing can be achieved successively towards different tracks, therefore addressing different trains and delivering specific data to these trains.

The autocorrelation function is used to evaluate the temporal focusing (TF) and the spatial focusing (SF). To study TF, one can evaluate the Focusing Gain (FG), which is defined as the ratio of the spectrum power of strongest amplitude peak in TR received to the strongest peak received by a conventional UWB system [22]. The focusing gain can be written as:

$F G_{[d B]}=20 \log _{10}\left(\frac{\max \left(\left|r_{r t}(t)\right|\right)}{\max (|y(t)|)}\right)$ 
Fig. 2 Principle of Time

Reversal technique

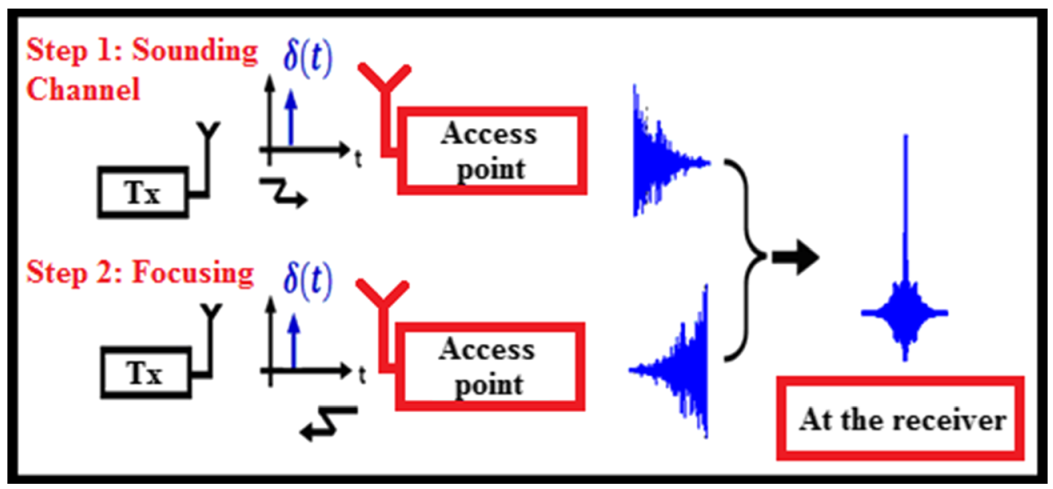

Higher FG could potentially translate into higher communication range and higher precision of localization for a localization system as compared to a classical UWB system.

The study of SF is performed considering a TR-UWB system in a SISO configuration. The channel impulse response (CIR) of the intended receiver which is located in position $p_{0}$ is noted $h\left(p_{0}, t\right)$. The CIR of the unintended receiver located in position $p_{i,(i \neq 0)}$ is noted $h\left(p_{i, i \neq 0}, t\right)$. The equivalent CIR of the intended receiver is then given by:

$h e q\left(p_{0}, t\right)=h^{*}\left(p_{0},-t\right) \otimes h\left(p_{0}, t\right)$

While the equivalent impulse response of the unintended receiver is given by:

$h e q\left(p_{i, i \neq 0}, t\right)=h^{*}\left(p_{0},-t\right) \otimes h\left(p_{i}, t\right)$

SF is then evaluated as the ratio of the strongest peak power received by the intended receiver to the strongest peak received by the unintended receiver [25]. The SF parameter can be written as:

$S F_{[d B]}=20 \log _{10}\left(\frac{\max \left(\left|h e q\left(p_{0}, t\right)\right|\right)}{\max \left(\left|h e q\left(p_{1}, t\right)\right|\right)}\right)$

\subsection{Channel Modeling (Deterministic Approach)}

The objective of this modeling is to characterize the channel impulse response using a deterministic approach. As stated above, TR would benefit from the complexity of the propagation environment. The more complex the environment, the better the focus of energy should be [26]. Therefore, we propose to select a channel model which allows this TR feature to be tested progressively using an increasing number of paths i.e. 2, 3, 4, 6 and 10 paths.
To model the channel, infinitesimally short dipoles or point sources are used for the transmitter and receiver antennas, and a deterministic approach is used. Two parallel infinite and flat dielectric walls are considered, corresponding to a floor and a ceiling of a generic environment shown in Fig. 3. $\mathrm{T}_{\text {he }}$ transmitter and the receiver are separated by $d_{0}$. Except for the direct path (LOS), the signals transmitted to the receiver undergo reflections on the floor and ceiling.

These reflections can be divided into two categories; the first reflection is created due to the floor surface, the second one is created due to the first reflection happening on the ceiling. It should be noted that these two cases have common rules. First, our interest will focus on the first reflection on the floor. It has been shown that this model, although established based on case 1 , is valid for case 2 as well by applying the principles of geometrical optics [27].

In Fig. 3, the number of paths corresponds to the number of reflections between the floor and the ceiling. The first path is the direct path $r_{0}$, the second path is the path reflected on the floor; its length corresponds to $r_{1}$. The third path is the one undergoing two reflections, one on the floor and one on the ceiling.

From this model, it is possible to obtain the length of all the paths linking $T_{x}$ and $R_{x}$, using the image method and the geometrical optical approach [28].

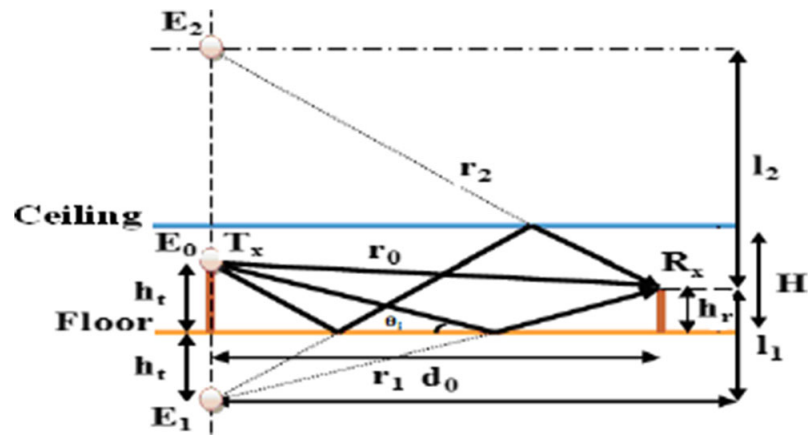

Fig. 3 Channel model configuration (image method) 
- $\quad$ The length of path $\mathrm{N}^{\circ} 0$ is given by:

$$
r_{0}=\sqrt{d_{0}^{2}+l_{0}^{2}} ; \text { With } l_{0}=\left|h_{t}-h_{r}\right|
$$

- $\quad$ The length of path $\mathrm{N}^{\circ} 1$ is given by:

$$
r_{1}=\sqrt{d_{0}^{2}+l_{1}^{2}} ; \text { With } l_{1}=\left|h_{t}+h_{r}\right|
$$

- $\quad$ The length of path $\mathrm{N}^{\circ} 2$ is given by:

$$
r_{2}=\sqrt{d_{0}^{2}+l_{2}^{2}} \text {; With } l_{2}=2 H+\left|h_{t}-h_{r}\right|
$$

- The length of path $\mathrm{N}^{\circ} \mathrm{n}$ is given by:

$$
\begin{aligned}
r_{n} & =\sqrt{d_{0}^{2}+l_{n}^{2}} ; \text { With } l_{n} \\
& =\left\{\begin{array}{l}
n H+\left|h_{t}-h_{r}\right| \text { if } \mathrm{n} \text { is even } \\
(n-1) H+\left|h_{t}+h_{r}\right| \text { if } \mathrm{n} \text { is odd }
\end{array}\right.
\end{aligned}
$$

$n$ corresponds to the path number or the number of reflections.

Consider that the additive white Gaussian noise (AWGN) $n(t)=0$. Thus, the received signal is given by:

$y(t)=y_{d}(t)+y_{r}(t)$

$y_{d}(t)=\left(\frac{\lambda}{4 \pi r_{0}}\right) s\left(t-\tau_{0}\right)$

$$
\begin{aligned}
y_{r}(t) & =\frac{\lambda}{4 \pi}\left(\sum_{n=1}^{N}\left[R_{v}\left(\theta_{n}\right)\right]^{n} \frac{s\left(t-\tau_{n}\right)}{r_{n}}\right) \\
& +\left(\sum_{n=1}^{N}\left[R_{h}\left(\theta_{n}\right)\right]^{n} \frac{s\left(t-\tau_{n}\right)}{r_{n}}\right)
\end{aligned}
$$

Where, $y_{d}(t)$ corresponds to the received signal with the direct path. $r_{0}$ and $\tau_{0}$ are respectively the distance travelled and the corresponding time of flight. $y_{r}(t)$ is the received signal constructed with the $N$ reflected paths. $\lambda$ is the wavelength. $R_{v}\left(\theta_{n}\right)$ and $R_{h}\left(\theta_{n}\right)$ are the reflection coefficients for vertical and horizontal polarizations; they are given by [29]:
$R_{v}\left(\theta_{n}\right)=\frac{\cos \theta_{n}-\sqrt{\frac{1}{\varepsilon_{r}}-\frac{1}{\varepsilon_{r}^{2}} \sin ^{2} \theta_{n}}}{\cos \theta_{n}+\sqrt{\frac{1}{\varepsilon_{r}}-\frac{1}{\varepsilon_{r}^{2}} \sin ^{2} \theta_{n}}}$

$R_{h}\left(\theta_{n}\right)=\frac{\cos \theta_{n}-\sqrt{\varepsilon_{r}-\sin ^{2} \theta_{n}}}{\cos \theta_{n}+\sqrt{\varepsilon_{r}-\sin \theta_{n}}}$

$\varepsilon_{r}$ is the relative permittivity of the floor or ceiling and $\theta_{n}$ is the incident angle of the path number $\mathrm{n}$. A conventional $\varepsilon_{r}=10$ value is used for concrete walls.

For different rays, reflections angle $\theta$ is different. It is given by:

$\theta_{n}=\arcsin \left(\frac{d_{0}}{r_{n}}\right)$

$$
r_{n} \text { is the distance travelled }\left(r_{n}=\sqrt{d_{0}^{2}+l_{n}^{2}}\right) \text {. }
$$

\section{Performances Evaluation}

\subsection{Analytical Study of TR Characteristics}

The study of the temporal focusing is based on the characterization of the propagation channel. We successively determine the equivalent CIR, the Power Delay Profile and corresponding Focusing Gain.

Throughout the study, we denote by:

- $\quad x(t)$ : the transmitted pulse (second derivative Gaussian pulse);

- $\quad c(t):$ the CIR;

- $\quad c^{*}(-t)$ : the conjugated and reversed CIR.

In this specific case, the determination of the focusing gain is obtained on the basis of the calculation of the channel impulse response.

The channel impulse response for conventional UWB-IR is given by:

$c(t)=\sum_{i=0}^{N-1} \alpha_{i} x\left(t-t_{i}\right)$

The Power Delay Profile is then determined directly by:

$\operatorname{PDP}_{U W B-I R}(t)=|c(t)|^{2}=\left|\sum_{i=0}^{N-1} \alpha_{i} x\left(t-t_{i}\right)\right|^{2}$ 
In turn the equivalent channel impulse response corresponding to the TR-UWB system is given by:

$\operatorname{ceq}(t)=c^{*}(-t) \otimes c(t)=\int_{A} c^{*}(t+\tau) c(\tau) d \tau$

And one also has:

$\operatorname{ceq}(t)=\int_{A} c(\tau) c^{*}(t+\tau) d \tau$

By replacing $c(t)$ by its expression, (21) becomes:

$c e q(t)=\int_{A} \sum_{i=0}^{N-1} \alpha_{i}^{2} x\left(\tau-t_{i}\right) x\left(t+\tau-t_{i}\right) d \tau=\sum_{i=0}^{N-1} \alpha_{i}^{2} \varphi_{i x}(t)$

where $\varphi_{i x}(t)=\int_{A} x\left(\tau-t_{i}\right) x\left(\tau-t_{i}+t\right) d \tau$ and $A=\left[0, T_{5}\right]$.

The Power Delay Profile is then given by:

$P D P_{T R-U W B}(t)=|\operatorname{ceq}(t)|^{2}=\left|\sum_{i=0}^{N-1} \alpha_{i}^{2} \varphi_{i x}(t)\right|^{2}$

The Appendix contains the case study of Focusing Gain for two paths SISO configuration and generalization to other cases using Eq. (4). Thus, the expression of FG is given below

$F G_{\text {SISOipaths }}=10 \log _{10}\left(\frac{\sum_{i=0}^{N-1} \alpha_{1}^{2}}{\alpha_{0}^{2}}\right)$

\subsection{Analytical and Simulation Results}

In this section, we compare the simulation and the analytical results for UWB and TR-UWB cases. Figure 4 shows
Table 1 Comparative study between analytical and simulation results in terms of Focusing Gain results

\begin{tabular}{lllll}
\hline Channels models & 2 paths & 4 paths & 6 paths & 10 paths \\
\hline $\mathrm{FG}_{\text {Analytical }}[\mathrm{dB}]$ & 2.60 & 5.30 & 5.63 & 5.82 \\
$\mathrm{FG}_{\text {simulation }}[\mathrm{dB}]$ & 2.97 & 5.40 & 5.92 & 6.27 \\
\hline
\end{tabular}

comparison results in the case of the SISO 2 path configuration. These results correspond to the equivalent channel impulse response $h e q(t)$ and Power Delay Profile $P D P_{T R-U W B}$. We observe that the simulation results are close to the analytical results. The same conclusions are available using SISO configurations with 4, 6 and 10 paths. Table 1 gives comparative results in terms of Focusing Gain (FG). These results confirm, TR takes advantage of the complexity of the channel. The gain obtained using the two paths SISO configuration for analytical and simulation study are $2.60 \mathrm{~dB}$ and $2.97 \mathrm{~dB}$ respectively. It increases to $5.82 \mathrm{~dB}$ and $6.30 \mathrm{~dB}$ for 10 paths.

In Fig. 5, the focusing gain versus number of paths reaches a horizontal asymptote after $8-10$ paths because the attenuation of the reflected signals becomes more and more important with the number of reflected paths. Thus, their corresponding contributions decrease.

To study spatial focusing (SF), we consider the SISO 4 paths configuration to calculate the equivalent impulse response ceq $\left(p_{0}, t\right)$ of the intended receiver corresponding to the reference distance $d_{0}=10 m$ between $\mathrm{T}_{\mathrm{x}}$ and $\mathrm{R}_{\mathrm{x}}$ (Fig. 6a). The equivalent impulse response $c e q\left(p_{1}, t\right)$ for the unintended receiver positioned in $P_{1}$, at $d_{1}=20 \mathrm{~m}$ is illustrated in Fig. 6b. Moving from $P_{0}$ to $P_{1}$, we obtain a loss of focus of more than $8 \mathrm{~dB}$.

\subsection{Contribution of TR-UWB System in Terms of Localization Accuracy}

In this section, the localization error is evaluated considering two different cases: UWB-IR alone and UWB-IR combined
Fig. 4 Analytical and simulation results for TR-UWB a $h e q(t), \mathbf{b}$ $P D P_{T R-U W B}(t)$

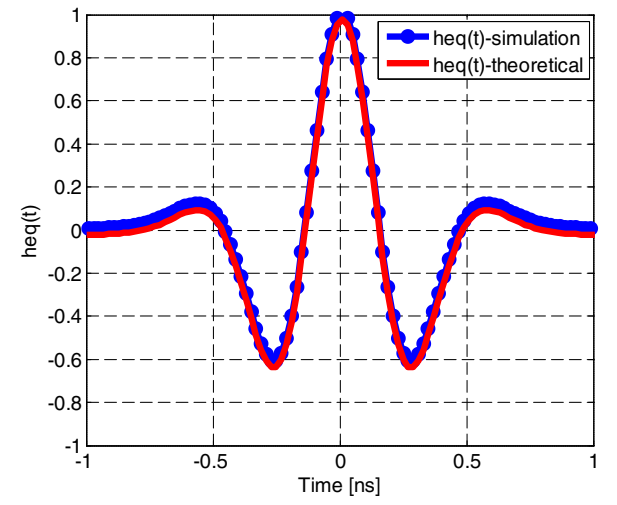

(a)

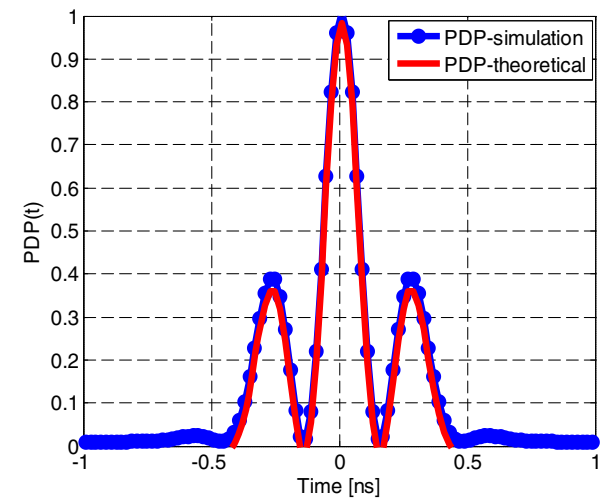

(b) 


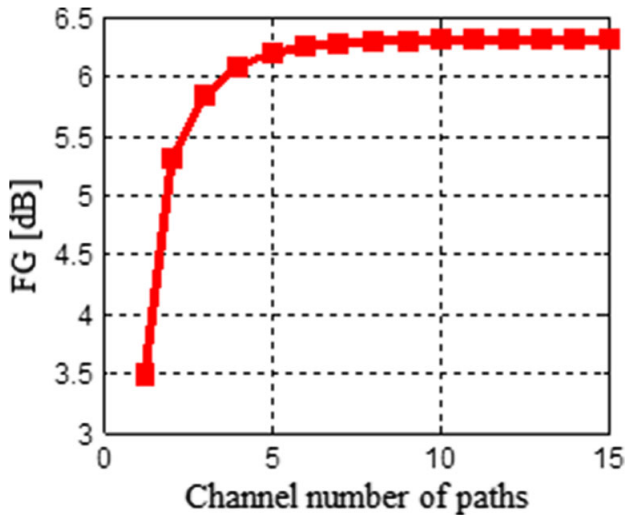

Fig. 5 Focusing gain vs. path number

with TR. The goal is to estimate the contribution of TR in terms of precision of localization as a function of the propagation environment complexity. Using three base stations, we determine the position of a mobile in a $2 \mathrm{D}$ plane. To locate the mobile, each base station sends its own signal (recorded and reversed in time in the case of TR). The UWB-IR signals are formed by second derivative Gaussian pulse that modulated by an antipodal modulation and coded by a Gold code [30], one code per base station. Processing is performed at the mobile $\left(R_{x}\right)$ to determine its position relative to the base stations. The distance error is given by the difference between the calculated and the actual position of the mobile.

The mobile receives the signals from each base station and performs an adequate signal processing to determine its position, relative to the base stations. Using the TDOA technique, the signal received at the mobile is processed to retrieve the position of the latter [31]. The Chan algorithm is used to solve the nonlinear equations imposed by the TDOA technique [32].

For the comparative study between the conventional localization system UWB-IR and the proposed TR_UWB system, we first use the UWB-IR case to locate the mobile then the obtained information on the position of the mobile is used as reference for locating with the TR-UWB system. Our comparison is based on the computation of the Root Mean Square
Error (RMSE) of localization between the conventional UWB-IR system and the proposed TR-UWB-IR system.

We consider the SISO configuration with 10 paths. An AWGN is also injected into the channel. Figure 7 shows the comparative study of the RMSE for both systems. A SNR of $3 \mathrm{~dB}$ is considered in each case. In these conditions, we obtain a better precision of localization using the TR-UWB-IR system.

Trying to confirm these results, we then repeat this operation with the SISO 2, 6 and 10 path channels. A large number of iterations are used (2000).

Table 2 presents the simulation results in these three cases. These results show that TR-UWB gives the best performance in terms of localization. Indeed, in the analysis case, the localization error is above $21.0 \mathrm{~cm}$ in the case of SISO 10 paths without TR, while it is only $1.30 \mathrm{~cm}$ with TR.

These preliminary results show that the combination of UWB and TR allows for a more accurate localization to be obtained, that could be in line with the decimetre necessary level of precision required by the railway beacon application.

The localization RMSE of versus SNR is then evaluated. The results are presented in Fig. 8.

The conventional UWB and TR-UWB results obtained for the 2, 6 and 10 paths configurations are very different with a significant advantage to the TR-UWB configurations. We note that for $\mathrm{SNR}>2 \mathrm{~dB}$, the localization error remains fairly low. The fairly high performance of the proposed system on simulated data can be probably explained by the optimal conditions used in the simulation scenario.

\section{Experimentation Measurements}

In this section, the characteristics of Time Reversal (Temporal Focusing and Spatial Focusing) and error localization are studied in the practical case. The experimentations are performed in an anechoic chamber.
Fig. 6 a The intended receiver $\operatorname{ceq}\left(p_{0, t}\right)\left(d_{0}=10 \mathrm{~m}\right), \mathbf{b}$ The unintended receiver ceq $\left(p_{1, t}\right)\left(d_{1}=\right.$ $20 m$ )

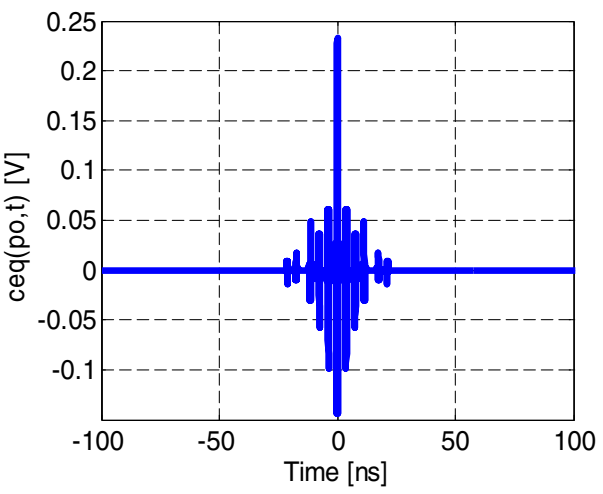

(a)

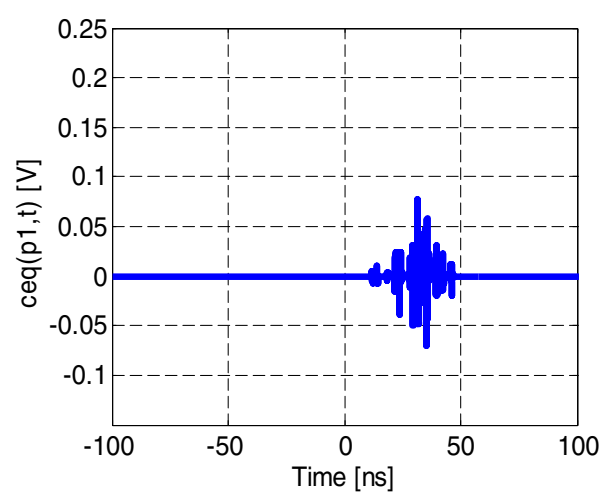

(b) 
Fig. 7 RMSE evaluation for conventional UWB-IR and TRUWB ( 2 D positioning system)

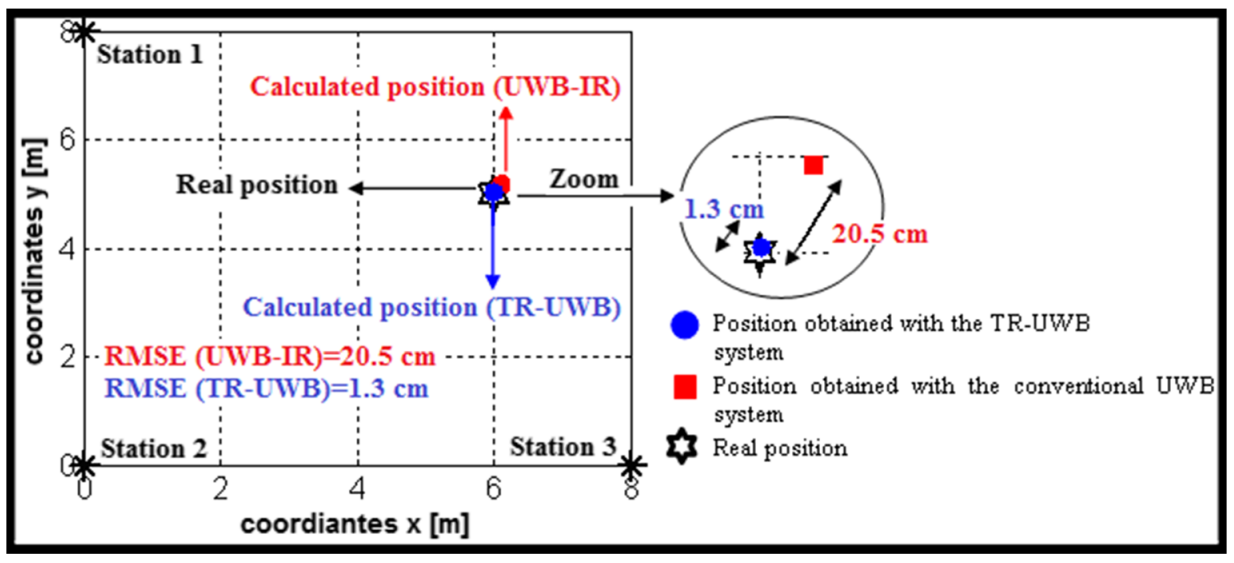

\subsection{TR Characteristics Evaluation}

An Arbitrary Waveform Generator (AWG) associated with a fast sampling oscilloscope (TDS) is used. Pulses are radiated using a wideband horn antenna. The input of a low noise amplifier (LNA) is connected to a receiving horn antenna similar to the transmitting antenna. The LNA output is connected to the input of the oscilloscope. A portable computer is used to process the signals and store the results. Measuring equipment is installed in an anechoic chamber. Large metallic plates are used to simulate corresponding reflectors.

The dimension of the anechoic chamber we used is $7 \times 7 \times$ $3 \mathrm{~m}$, it is operating from $100 \mathrm{MHz}$ to $10 \mathrm{GHz}$.

Two antennal configurations are considered:

- A SISO configuration consists of a single transmitting antenna and a single receiving antenna;

- A MISO 3x1 configuration consists of three transmitting antennas and one receiving antenna.

For each type of configuration, three cases are considered:

1 two reflector plates are introduced to increase the number of reflected signals;

2 three plates are installed to further increase the number of reflections in the propagation environment;

Table 2 Comparative analysis between the UWB impulse radio (UWB-IR) and TR-UWB in terms of focusing gain and root mean square error localization (SISO 2,6 and 10 path configuration, SNR= $3 \mathrm{~dB})$

\begin{tabular}{llll}
\hline SISO Config. & FG $_{[\mathrm{dB}]}$ & RMSE UWB-IR $_{[\mathrm{cm}]}$ & RMSE TR-UWB $_{[\mathrm{cm}]}$ \\
\hline 2 paths & 2.97 & 19.35 & 0.43 \\
6 paths & 6.27 & 19.65 & 0.80 \\
10 paths & 6.30 & 20.50 & 1.30 \\
\hline
\end{tabular}

3 four plates are present to maximize the propagation environment complexity. Figure 9 depicts the specific two reflectors scenario (SISO).

For each experimental configuration we determine the corresponding Focusing Gain. As presented in section II, a UWB pulse is first transmitted from Tx to Rx using a selected reflector configuration. This received signal is recorded and reversed in time. The reversed signal is then delivered to the arbitrary waveform generator. Secondly, the reversed signal is generated and transmitted from Tx to Rx. In the cases of MISO $3 \times 1$, each Tx re-emits its corresponding reversed in time signal.

\subsection{Experimental Results}

Figures 10 and 11 show the equivalent channel impulse response without TR and with TR in the case of the two reflector configuration respectively. The direct path and following reflected paths can be observed in Fig. 10.

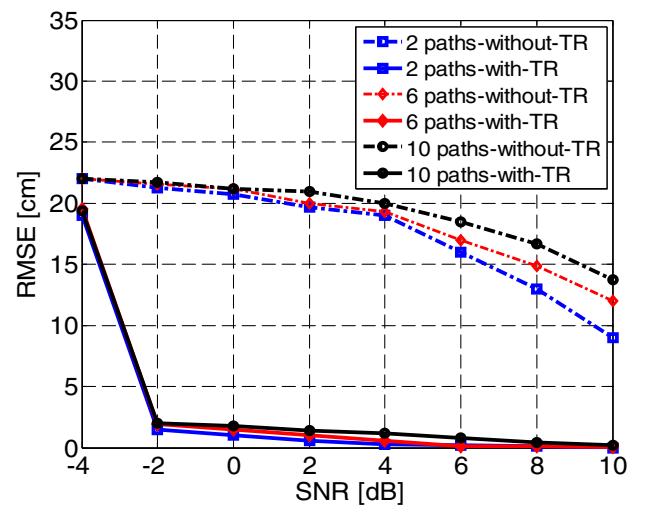

Fig. 8 Comparison UWB-IR vs. UWB-IR/TR, RMSE vs. SNR for SISO 2, 6 and 10 paths 


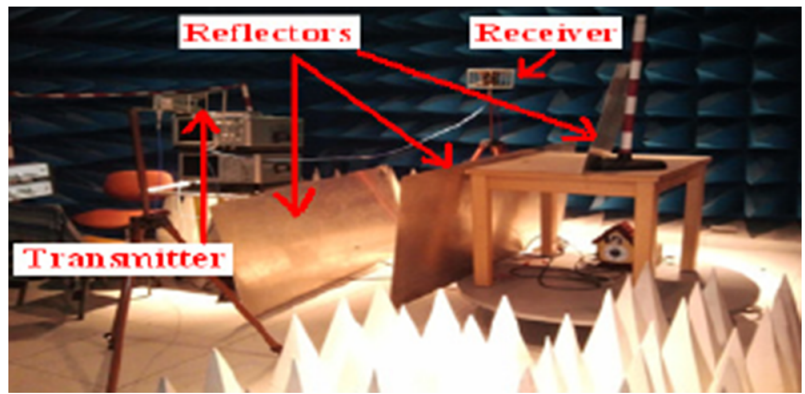

Fig. 9 Experimental setup

Table 3 shows the results in terms of FG for the 2, 3 and 4 reflector configurations (SISO and MISO $3 \times 1$ configuration). As expected, in the case of SISO configuration, FG increased from $3.2 \mathrm{~dB}$ with two reflector plates up to $6.14 \mathrm{~dB}$ using four reflector plates. Moreover, by comparing the values of FG for these three types of configuration, we find that FG increases from SISO to MISO configuration. Considering the four reflector scenario, FG is $6.14 \mathrm{~dB}$ in the SISO configuration, increasing to $12.8 \mathrm{~dB}$ in the MISO $3 \times 1$ configuration. This result confirms that the performance of TR takes advantage of the complexity of channel.

The Spatial Focusing is evaluated using three reflectors (Configuration 3). Moving the receiver at position $\mathrm{p}_{1}$ corresponds to $50 \mathrm{~cm}$ from its initial position $\mathrm{p}_{0}$ (Fig. 12) causes a loss of focus of more than $10 \mathrm{~dB}$ as shown in Fig. 13.

\subsection{Localization Error Evaluation}

In this last section, we perform a comparative evaluation between a conventional UWB positioning and a TR-UWB positioning system. To compute the $2 \mathrm{D}$ receiver position information, we need at least three transmitters. Thus, we consider our previous MISO $3 \times 1$ configuration. The operating principle is as follows:

- In the case of the UWB alone system, a sequence of 7 pulses is transmitted by each station to the receiving

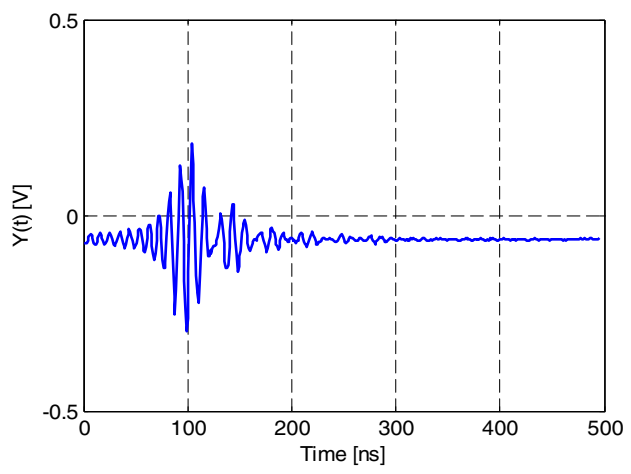

Fig. 10 Received signal (UWB-IR) in the experimental setup

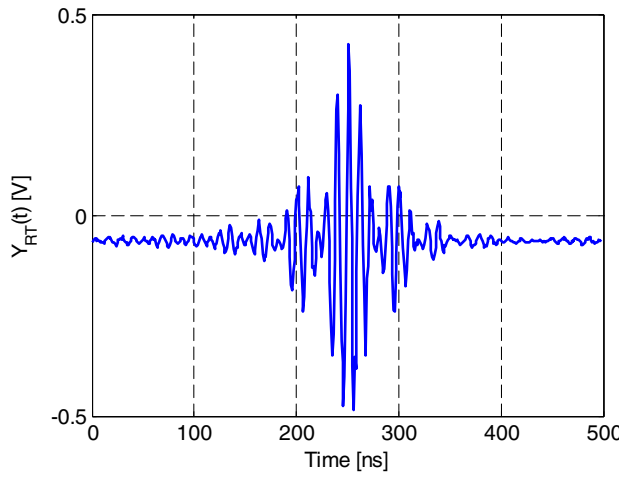

Fig. 11 Received signal (TR-UWB) in the experimetal setup

station. Each transmitter has its own coded signal. The received signals are acquired by the TDS. Post processing evaluates the arrival times of the various signals using a Time Difference of Arrival algorithm (TDOA). Then, the Chan localization algorithm is applied to determine the receiver position [32].

- $\quad$ For the TR-UWB system, each transmitting station sends first a signal which is acquired by the oscilloscope and respectively sent back to the transmitters. The time reversed signals are transmitted from each base station to the receiver. The received signals are then acquired in this TR condition. Then, the same post processing than in the UWB alone system determines the receiver position. However, in this TR configuration, the received signals are correlated with their TR reference signals, and not with the initial UWB sequence.

Experiments were also carried out successively in the anechoic chamber and in the selected indoor environment.

Three series of acquisition were performed. Figure 14a and b show an example of the received signals from Tx1 using three reflector plates respectively without TR and with TR. As previously mentioned, a sequence of seven pulses is sent.

After processing, we determine the position of the mobile. Figure 15 shows the corresponding position errors. With the conventional UWB localization system, we get an error of $11.0 \mathrm{~cm}$, decreasing to $3.3 \mathrm{~cm}$ in the case of TR-UWB.

To study the performance of the two systems in our various scenarios, we systematically determined position errors for both UWB and TR-UWB systems. To do this, three sets of acquisition

Table 3 Focusing gain (experimental results)

\begin{tabular}{llc}
\hline Configuration & SISO & MISO 3×1 \\
\hline $\mathrm{FG}_{[\mathrm{dB}]}$ (2 reflectors) & 3.2 & 8.2 \\
$\mathrm{FG}_{[\mathrm{dB}]}$ (3 reflectors) & 5.1 & 9.4 \\
$\mathrm{FG}_{[\mathrm{dB}]}$ (4 reflectors) & 6.14 & 12.8 \\
\hline
\end{tabular}


Fig. 12 Principle of SF

measurement

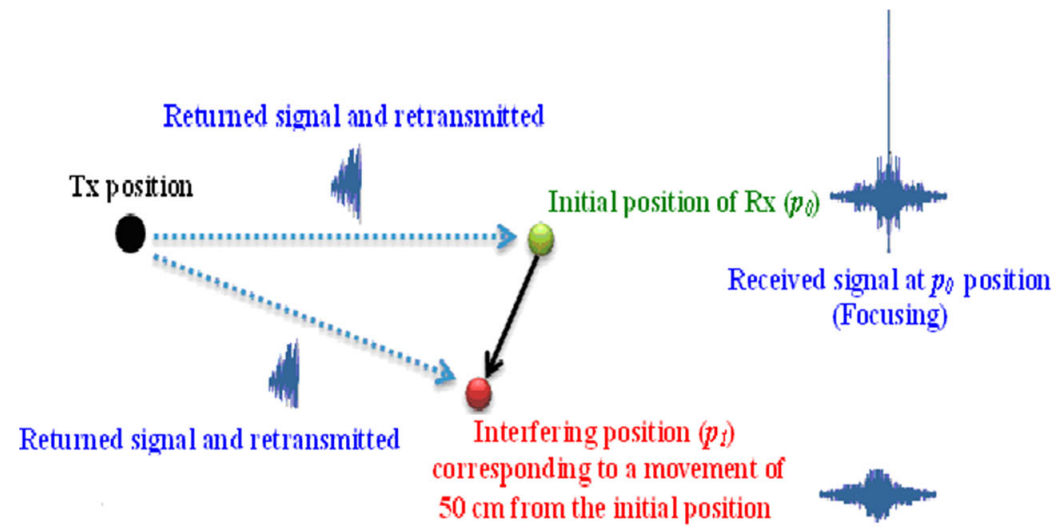

Received signal at $p_{1}$ position

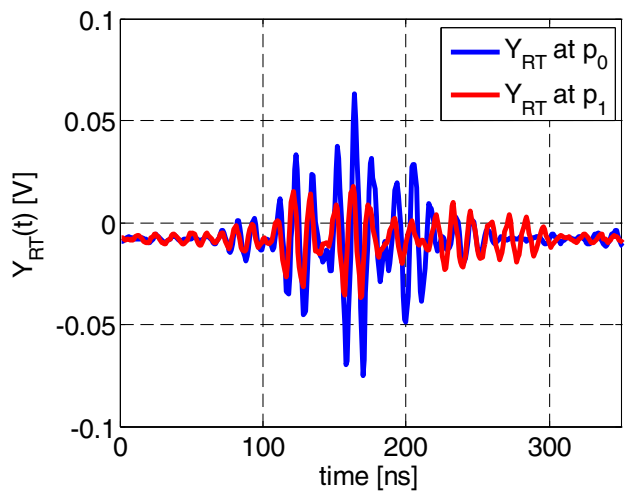

Fig. 13 Received signals at $\mathrm{p}_{0}$ and $\mathrm{p}_{1}$ by evaluating Spatial Focusing SF

have been exploited and processed and position errors were determined. The results are reported in Tables 4 and 5.

The average values obtained for the three acquisitions are reported in Tables 6 and 7. Comparing the two systems in the anechoic chamber, without reflector, leads to little difference. Even if the TR-UWB system provides a lower error on localization accuracy, the difference is fairly limited. Of course in a non multipath environment like the anechoic chamber, TR adds little improvement. For all the other considered cases, TR-UWB significantly provides better localization accuracy.

\section{Conclusion and Future Work}

In this contribution, results obtained working on the association of Time Reversal and UWB impulse radio for a localization application is presented. Channel propagation models are based on a deterministic channel model approach. The analytical, simulation and experimental results have shown that this particular combination can reduce the error localization thanks to the focusing characteristics of time reversal. To validate the analytical and simulation results, experimentations were conducted in anechoic chamber. Future work will consider a real railway balise environment (using trains), as well as the realization of a balise prototype. The localization error and communication performances will be also evaluated by the tests in practical case.
Fig. 14 a Received signal in the case of conventional UWB localization system (without TR) b Received signal in the case of TR-UWB localization system

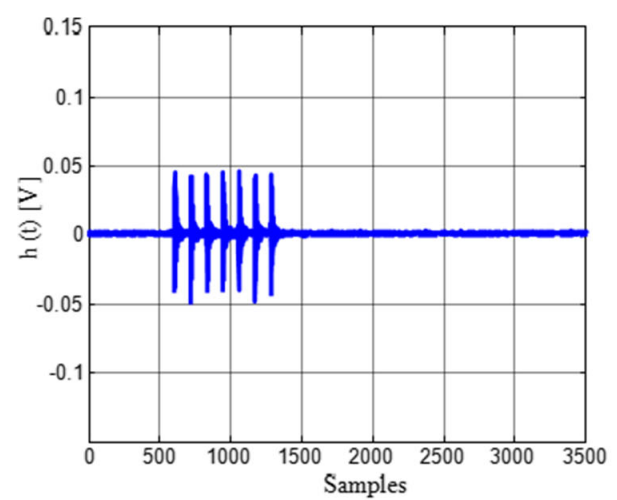

(a)

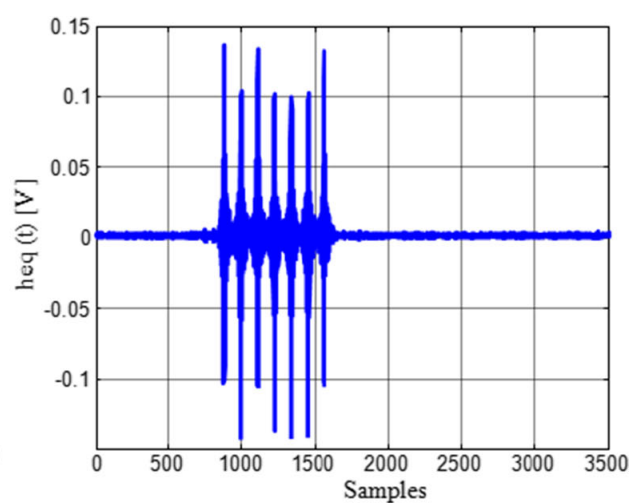

(b) 
Fig. 15 Conventional UWB and

TR-UWB localization systems

(Scenario with 3 reflector plates in

an anechoic chamber)

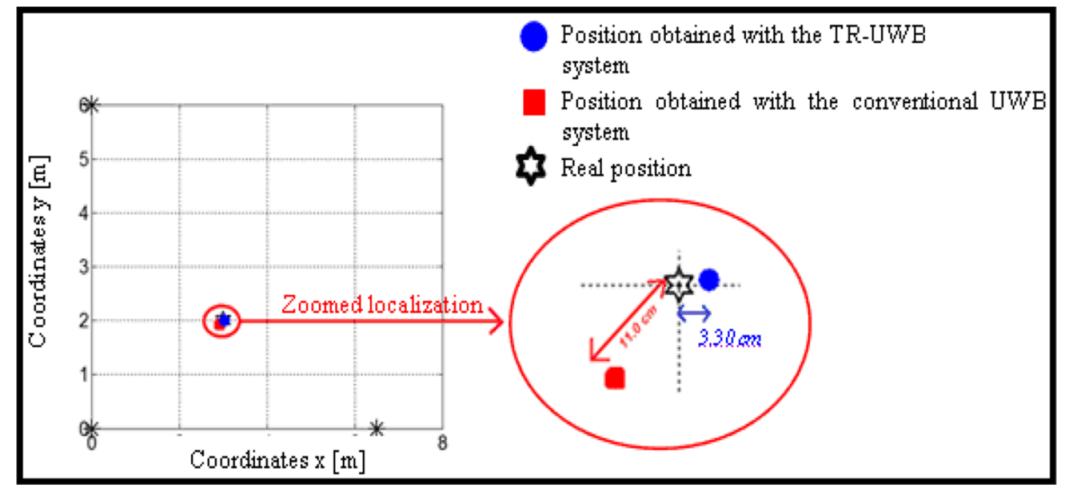

\begin{tabular}{lllll}
\hline & Configuration & First set & Second set & Third set \\
\hline Position error $[\mathrm{cm}]$ & 2 reflectors & 10.9 & 11.1 & 11.3 \\
& 3 reflectors & 12.2 & 12.1 & 12.1 \\
& 4 reflectors & 13.2 & 13.3 & 13.4 \\
\hline
\end{tabular}

Table 4 Position errors, UWB Alone
Table 5 Position errors, TRUWB

\begin{tabular}{llllr}
\hline & Configuration & First set & Second set & Third set \\
\hline Position error $[\mathrm{cm}]$ & 2 reflectors & 5.2 & 5.4 & 5.3 \\
& 3 reflectors & 3.9 & 4.0 & 4.1 \\
& 4 reflectors & 3.5 & 3.6 & 3.5 \\
\hline
\end{tabular}

Table 6 Average position errors considering the 3 acquisitions, UWB alone

\begin{tabular}{llll}
\hline Number of reflectors & 2 & 3 & 4 \\
\hline Position error [cm] & 11.1 & 12.1 & 13.3 \\
\hline
\end{tabular}

Table 7 Average localization errors considering the 3 acquisitions, TRUWB

\begin{tabular}{llll}
\hline Number of reflectors & 2 & 3 & 4 \\
\hline Position error [cm] & 5.3 & 4.0 & 3.5 \\
\hline
\end{tabular}




\section{Appendix}

Focusing computation for SISO Configuration channel model

For SISO 2 paths model, the equivalent channel impulse response is given by:

$c e q(t)=\sum_{i=0}^{1} \alpha_{i}^{2} \varphi_{i x}(t)=\alpha_{0}^{2} \varphi_{0 x}(t)+\alpha_{1}^{2} \varphi_{1 x}(t)$

The Power Delay Profile is the given by:

$$
P D P_{T R-U W B}(t)=\left|\alpha_{0}^{2} \varphi_{0 x}(t)+\alpha_{1}^{2} \varphi_{1 x}^{2}(t)\right|^{2}
$$

$\alpha_{i}$ is composed of the reflection coefficients and the reflected path length

The peak of $P D P_{U W B}(t)$ is obtained at $t_{0}$, then:

$$
\max \left(P D P_{U W B-I R}(t)\right)=\alpha_{0}^{2}
$$

Also, the peak of $\left(P D P_{T R-U W B}(t)\right)$ is obtained at $\mathrm{t}=0$, then

$\max \left(P D P_{T R-U W B}(t)\right)=\sum_{i=0}^{N-1} \alpha_{i}^{2}$

Therefore:

$F G_{\text {SISOipaths }}=10 \log _{10}\left(\frac{\sum_{i=0}^{N-1} \alpha_{i}^{2}}{\alpha_{0}^{2}}\right)$

Then for SISO 2 paths:

$$
F G_{\text {SISO2paths }}=10 \log _{10}\left(1+\frac{\alpha_{1}^{2}}{\alpha_{0}^{2}}\right)
$$

\section{References}

1. Saghir, H., Heddebaut, M., Elbahhar, F., Rouvaen, J.M., Rivenq, A.: "Train-to-wayside wireless communication in tunnel using ultrawideband and time reversal ," Ed. Elsevier, Transportation Research Part C: Emerging Technologies vol. 17, No. 1, 2009, pp. 81-97

2. José, A., Santos, D., Soares, A.R., Redondo, F.M.A., Carvalho, N.B.: Tracking trains via radio frequency systems. IEEE Trans. Intell. Trans. Syst 6(2), 244-258 (2005)

3. Fararooy, S., Allan, J., Jin, J., Maleki, K., Pang, F.: “Accurate Train Localization In Open Space And Tunnels," International Conference on Public Transport Electronic Systems, Institution of Electrical Engineers, London, UK, 21-22 May 1996, pp. 25-29
4. International Union of Railways, "ERTMS - European Rail Traffic Management System," http:/www.uic.org/spip.php?rubrique847

5. Plan, O.: "GIS-gestutzte Verfolgung von Lokomotiven im Werkbahnverkehr," Ph.D. dissertation, University of German Federal Armed Forces Munich, Germany, (2003)

6. Zwirello, L., Schipper, T., Harter, M., Zwick, T.: "UWB Localization System for Indoor Application: Concept, Realization and Analysis," Journal of Electrical and Computer Engineering, Article ID 849638, 2012, $11 \mathrm{p}$

7. Lerosey, G.: "Retournement Temporel d'ondes Electromagnétiques et Application à la Télécommunication en Milieux Complexes," Phd thesis, Paris 7 University- Dénis Diderot, 2006

8. Benslimen, M.N.: "Recherche de procedure de caractérisation de l'Environnement Elecromagnétique Ferroviaire Adaptées au contexte des systèmes de communications Embarqués," Ph.D. thesis, Lille University (France), 2009

9. Maaref, N., Millot, P., Ferriéres, X., Pichot, C., Picon, O. Electromagnetic imaging methode based on time reversal processing applied to through the wall target localization. Prog. Electromagn. Res. M 1, 59-67 (2008)

10. Molish, A.F.: Ultrawideband propagation channel-theory, measurement, and modelling. Veh. Technol. IEEE Trans 54(5), 1528-1545 (2005)

11. Welbom, M.L.: "System Considerations for Ultra-Wideband Wireless Networks," IEEE: Radio and Wireless Conference RAWCON2001,Boston, Aug 19-22. 2001, pp.5-8

12. Saghir, H., Heddebaut, M., Elbahhar, F., Rivenq, A., Rouvaen, J.M. Time reversal UWB wireless communication-based train control in tunnel. J. Commun 4(4), 248-256 (2009)

13. Suwansantisuk, W., Win, M.Z., Shepp, L.A.: On the performance of wide-bandwidth signal acquisition in dense multipath channels. IEEE Trans. Veh. Technol. 54(5), 1584-1594 (2005)

14. Suwansantisuk, W., Win, M.Z.: Multipath aided rapid acquisition: optimal search strategies. IEEE Trans. Inform. Theory 53, 174-193 (2007)

15. Fink, M.: "Ondes et renversement du temps," Bulletin de l'union des professeurs de physique et de chimie, pp. 25-31 (2005)

16. Fink, M.: "Time Reversal waves and super resolution," Journal of Physics : Conference series 124. 4th AIP international conference and the 1 st congress of the IPIA, (2008)

17. Derode, A., Roux, P., Fink, M.: Robust acoustic time reversal with high order multiple scattering. Phys. Rev. Lett. 75(23), 4206-4209 (1995)

18. Van Atta, L.G.: “Electromagnetic Reflector," U.S. Patent 2908002 , Oct. 6, (1959)

19. Henty, B.E., Stancil, D.D.: Multipath-enabled super-resolution for $\mathrm{RF}$ and microwave communication using phase-conjugate arrays. Phys. Rev. Lett 93(24), 1-4 (2004)

20. Lerosey, G., de Rosny, J., Tourin, A., Derode, A., Montaldo, G., Fink, M.: Time reversal of electromagnetic waves. Phys. Rev. Lett. 92(19), 1-3 (2004)

21. Liu, X., Wang, B.-Z., Xiao, S., Deng, J.: Performance of impulse radio UWB communication based on time reversal technique. Prog. Electromagn. Res. PIER 79(11), 401-413 (2008)

22. Abassi-Moghadam, D., Tabataba Vakili, D.: Channel characterization of time reversal UWB communication systems. Wiley Int. J. Commun. Sys 65(9-10), 601-614 (2010)

23. Strohmer, T., Emami, M., Hansen, J., Papanicolaou, G., Paulraj, A. Application of Time-reversal with MMSE Equalizer to UWB Communications, pp. 3123-3127. Proceedings of Globecom Conference, Dallas (2004)

24. Fall, B., Elbahhar, M.F., Heddebaut, M., Rivenq, A.: "Time Reversal and UWB techniques for positioning system in transport applications, "Internation Symposium on Signal, Image, Video and Communications (ISIVC), University of Valenciennes (France), July 4-6 2012, pp.1-4

25. El-Salabi, H., Kiritsi, P., Paulraj, A., Papanicolaou, G.: "Experimental Investigation of Time Reversal Precoding for Space-Time Focusing 
in Wireless Communications," Supported by ONR grant Number N00014-02-1-0088

26. Naqvi, I.H.: “Application of Time Reversal (TR) Technique to Ultra WideBand (UWB) and multi antenna (MIMO) communication systems," Phd thesis, Institut National des Sciences Appliquées de Rennes, (2009)

27. Zhou, C.: "Impulsive Radio Propagation and Time Reversal MIMO System for UWB Wireless Communications," Ph.D. thesis, Faculty of the Graduated School Tennessee Technological University, (2008)

28. Mahmoud, S.F., Wait, J.R.: Geometrical optical approach for electromagnetic wave propagation in rectangular mine tunnels. Radio Sci. 9(12), 1147-1158 (1974)

29. Tanchotikul, S., Supannakoon, P., Promwong, S., Takada, J.: "RMS Delay Spread Estimation of Ground Reflection Channel for UWB Communications," International Symposium on Communication and Information Technologies (ISCIT), pp.1080-1083, (2005)

30. Elbahhar, F., Heddebaut, M., Rivenq, A., Rouvaen, J.M.: "Indoor positioning system based on the UWB technique," IPIN 2011, 21-23 September 2011, Guimarães, Portugal

31. Ghavami, M., Michael, L.V., Kohno, R.: Ultra Wide Band Signals and Sytems in Communication engineering. Ed. Wiley. London, 2007, $247 \mathrm{p}$

32. Chan, Y.T.: "A Simple and Efficient Estimator For Hyperbolic Location,” IEEE Transactions on Signal Processing, 1994

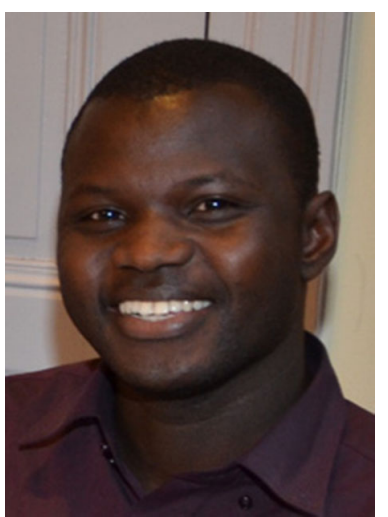

Bouna Fallwas born in 1983. He received the M.S and Ph.D. degrees from the University of Valenciennes (France) in 2009 and 2013, respectively. Currently he is assistant research at this university. His primary interest is in signal processing especially Ultra Wideband technology and Time reversal techniques applied to communication and localization systems.

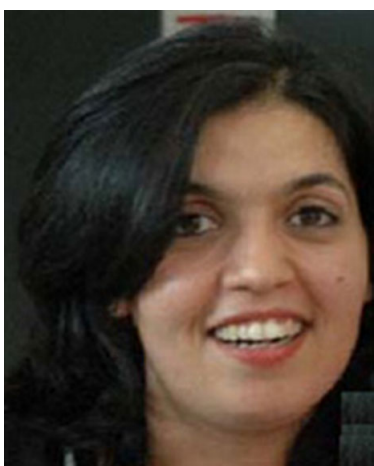

Fouzia Elbahhar was born in 1975. She received the M.S and Ph.D. degrees from the University of Valenciennes (France) in 2000 and 2003, respectively. She is now employed as researcher at IFSTTAR/LEOST, Villeneuve d'Ascq, France. She is involved in signal processing especially Ultra Wideband technology. Her major research interests are signal processing localization and communication techniques especially for land transportation like communication Vehicle to Vehicle and vehicle to infrastructure and localization systems.

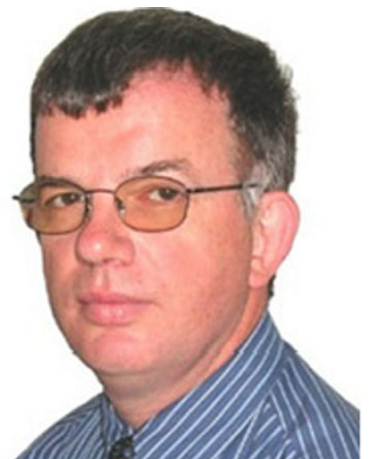

Marc Heddebaut was born In Somain, France, in 1955. He received the M.S. and Ph.D. degrees in electronics from the University of Lille France in 1980 and 1983, respectively. He joined the French National Institute for Transportation and Safety Research (INRETS now IFSTTAR) in 1983 and became A senior researcher in 1988. Since 1979, he has been working in the field of land mobile communication and electromagnetic compatibility. His primary interests include telecommunication and radar systems dedicated to land transport, EMC, mobile localization and command control of automated vehicles.

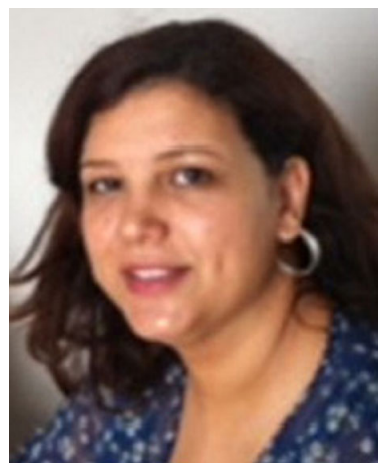

Atika Rivenq-Menhaj was born in 1970. She received her Diploma of Engineering and the M.S j degree in 1993 and then her Ph.D. degree in 1996, from the University of Valenciennes (France). She is now Professor in electronics at this university. Her primary interest is in signal processing applied to intelligent transportation systems and telecommunication systems

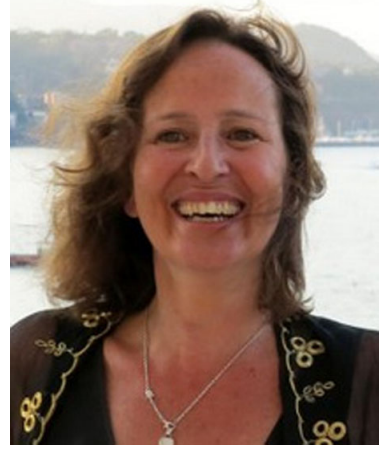

Maria Gabriella Di Benedetto Prof. Maria-Gabriella D Benedetto obtained her $\mathrm{Ph} . \mathrm{D}$. in Telecommunications in 1987 from the University of Rome La Sapienza, Italy. In 1991, she joined the Faculty of Engineering of University of Rome La Sapienza, where currently she is a Full Professor of Telecommunications at the INFOCOM Department. Her research interests include wireless communication systems and speech science: Ultra Wide Band, multi-carrier communications, and the introduction of cognitive principles in the radio and in the network 